\title{
On relations between gradient and classical equivariant homotopy groups of spheres
}

\author{
Kazimierz Gȩba and Marek Izydorek
}

\begin{abstract}
We investigate relations between stable equivariant homotopy groups of spheres in classical and gradient categories. To this end, the auxiliary category of orthogonal equivariant maps, a natural enlargement of the category of gradient maps, is used. Our result allows for describing stable equivariant homotopy groups of spheres in the category of orthogonal maps in terms of classical stable equivariant groups of spheres with shifted stems. We conjecture that stable equivariant homotopy groups of spheres for orthogonal maps and for gradient maps are isomorphic.
\end{abstract}

Mathematics Subject Classification. Primary 55P91; Secondary 55R70.

Keywords. Equivariant gradient maps, stable homotopy groups, fiberwise maps.

\section{Introduction}

Let $V$ be a finite-dimensional representation of a compact Lie group $G$. Consider a continuous $G$-equivariant map $f: U \rightarrow V$ defined on an open invariant subset $U \subset \mathbb{R}^{k} \oplus V$ such that $f^{-1}(0)$ is compact. It is known that in the equivariant homotopy class of $f$ there is a proper map. Such a map can be continuously extended to $\hat{f}: S^{k+V} \rightarrow S^{V}$, where spheres are onepoint compactifications of representations $\mathbb{R}^{k} \oplus V$ and $V$, respectively. Consequently, the homotopy class $[f]$ defines an element in the stable equivariant homotopy group $\omega_{k}^{G}\left(S^{0}\right)=\left\{S^{k+V} ; S^{V}\right\}_{G}^{*}$. The map $f: U \rightarrow V$ can be equipped with an additional structure. Assume that it is a gradient map; i.e., $f(x, v)=\nabla_{v} \varphi(x, v)$, where $\varphi: U \rightarrow R$ is an invariant $C^{1}$-function. If $\operatorname{dim} G>0$, then the homotopy of equivariant gradient maps is essentially more rigid than the ordinary equivariant homotopy. In particular, two gradient equivariant maps may represent the same equivariant homotopy class and may not be homotopic in the category of equivariant gradient maps. This phenomenon has been used by Dancer in [6]. He constructed a topological 
degree for $S^{1}$-equivariant gradient maps and used it to obtain a global bifurcation theorem applicable to cases where classical results give only limited information. This abstract result has been next applied to obtain a bifurcation theorem for periodic solutions of Hamiltonian systems, for a problem in elasticity and for elliptic equations on domains that admit $S^{1}$-symmetry. In fact our work is motivated by [6].

The aim of this paper is better understanding the homotopy-theoretical background of topological invariants associated with equivariant and gradient equivariant maps. More precisely, we investigate relations between gradient and nongradient equivariant homotopy groups of spheres. When trying to understand these relations it is convenient to consider yet another class - the class of orthogonal equivariant maps. That class is in some sense natural enlargement of the class of gradient equivariant maps. We are concerned with decomposition results in categories of equivariant maps, orthogonal equivariant maps and gradient equivariant maps. Our result, Theorem 1.3, allows for describing stable equivariant homotopy groups of spheres in the category of orthogonal maps in terms of classical stable equivariant groups of spheres with shifted stems. We conjecture that stable equivariant homotopy groups of spheres for orthogonal maps and for gradient maps are isomorphic. We would like to point out that methods developed in this paper allow for a simultaneous proof of the decomposition result (Theorem 1.1) in all three categories of mappings under consideration. The concept of otopy, introduced by Becker and Gottlieb in [3], provides a convenient framework for our proofs.

In what follows, every subgroup $H$ of a compact Lie group $G$ is understood to be closed and $(H)$ stands for a conjugacy class of $H$. If $N H$ is the normalizer of $H$ in $G$, then the quotient group $W H=N H / H$ is called the Weyl group (see [13]). Throughout the paper, $\mathbb{R}^{k}$ denotes the trivial representation of $G$ and $I$ is the unit interval $[0,1]$ with the trivial action of $G$. Finally, we set

$$
\begin{aligned}
\Phi(G) & =\{(H) ; H \text { is a closed subgroup of } G\}, \\
\Phi_{q}(G) & =\{(H) \in \Phi(G) ; \operatorname{dim} W H=q\} .
\end{aligned}
$$

We will be concerned with the following three families of maps.

(1) $\mathcal{M}_{G}^{*}\left(S^{k+V}, S^{V}\right)$ - the space of $G$-equivariant maps from $S^{k+V}$ into $S^{V}$ preserving a basepoint. $\left[S^{k+V}, S^{V}\right]_{G}^{*}$ is a set of corresponding homotopy classes.

(2) $\mathcal{M}_{G}^{\perp}\left(S^{k+V}, S^{V}\right)$ - the subspace of $\mathcal{M}_{G}^{*}\left(S^{k+V}, S^{V}\right)$ consisting of orthogonal $G$-maps. We say that $f \in \mathcal{M}_{G}^{*}\left(S^{k+V}, S^{V}\right)$ is a $G$-equivariant orthogonal map if

$$
f(x, v) \perp T_{v} G v
$$

for each $(x, v) \in f^{-1}(V) \subset \mathbb{R}^{k} \oplus V$. The set of corresponding homotopy classes is denoted by $\left[S^{k+V}, S^{V}\right]_{G}^{\perp}$.

(3) $f \in \mathcal{M}_{G}^{\nabla}\left(S^{k+V}, S^{V}\right)$ - the subspace of $\mathcal{M}_{G}^{*}\left(S^{k+V}, S^{V}\right)$ consisting of gradient $G$-maps. We say that $f \in \mathcal{M}_{G}^{*}\left(S^{k+V}, S^{V}\right)$ is a $G$-equivariant gradient map if there are a $G$-invariant open subset $U \subset f^{-1}(V), f^{-1}(0) \subset U$, 
and a $G$-map $\varphi: U \rightarrow \mathbb{R}$ such that

$$
\nabla_{v} \varphi(x, v)=f(x, v), \quad(x, v) \in U \subset \mathbb{R}^{k} \oplus V .
$$

$\left[S^{k+V}, S^{V}\right]_{G}^{\nabla}$ is a set of homotopy classes of equivariant gradient maps For an arbitrary $G$-representation $X$, a linear subspace $X^{H}$ of $X$ consisting of points fixed by $H$ is a representation of the Weyl group $W H$.

The isotropy type of an invariant subset $\Omega$ of a representation $X$ is the set

$$
\operatorname{Iso}(\Omega):=\left\{(H) \in \Phi(G) ; \Omega_{(H)} \neq \emptyset\right\} .
$$

Here we follow the terminology of [4]. Accordingly, $\operatorname{Iso}(\Omega)$ is partially ordered, $(H) \leq(K)$, if and only if $H$ is conjugate to a subgroup of $K$. The free isotropy type will be denoted by $(e)$ instead of $(\{e\})$. In particular, $X_{e}$ denotes a subset of $X$ on which $G$ acts freely. Assume that $(e) \in \operatorname{Iso}(V)$. Put $A^{k+V}=$ $S^{k+V} \backslash S_{e}^{k+V}$. We will also consider the maps $f \in \mathcal{M}_{G}^{\circ}\left(S^{k+V}, S^{V}\right)$ that send $A^{k+V}$ to a basepoint. The set of corresponding relative homotopy classes is denoted by $\left[S^{k+V}, A^{k+V} ; S^{V}, *\right]_{G}^{\circ}$, where $\circ=*, \perp$ or $\nabla$.

Our first result states as follows.

Theorem 1.1. There is a natural bijection

$$
\left[S^{k+V} ; S^{V}\right]_{G}^{\circ} \cong \prod_{(H) \in \operatorname{Iso}(V)}\left[S^{k+V^{H}}, A^{k+V^{H}} ; S^{V^{H}}, *\right]_{W H}^{\circ} .
$$

In the case $k+\operatorname{dim} V^{G} \geq 2$, this is an isomorphism of abelian groups:

$$
\left[S^{k+V} ; S^{V}\right]_{G}^{\circ} \cong \bigoplus_{(H) \in \operatorname{Iso}(V)}\left[S^{k+V^{H}}, A^{k+V^{H}} ; S^{V^{H}}, *\right]_{W H}^{\circ},
$$

where o means $*, \perp$ or $\nabla$.

The case $\circ=*$ is well known. One can find corresponding results in [8], [10], [11] and [12] and even more general theorems in [9]. However, our approach is different and works in each of the above three cases with analogous proofs.

Let $\left[S^{X} ; S^{Y}\right]_{G}^{\circ}$ be a set of homotopy classes of maps in one of the categories $\circ=*, \perp$ or $\nabla$.

The smash product with the identity map $\cdot \wedge\left(\mathrm{id}: S^{\mathbb{R}^{n}} \rightarrow S^{\mathbb{R}^{n}}\right)$ induces the suspension homomorphism

$$
\mathfrak{S}_{n}^{\circ}:\left[S^{X} ; S^{Y}\right]_{G}^{\circ} \longrightarrow\left[S^{X+n} ; S^{Y+n}\right]_{G}^{\circ} .
$$

If we let $X=\mathbb{R}^{k} \oplus V$ and $Y=V$, the stable homotopy group $\left\{S^{k+V} ; S^{V}\right\}_{G}^{\circ}$ is defined in a standard way.

The following theorem is an immediate consequence of Theorem 1.1.

Theorem 1.2. There is a natural isomorphism of abelian groups

$$
\left\{S^{k+V} ; S^{V}\right\}_{G}^{\circ} \cong \bigoplus_{(H) \in \operatorname{Iso}(V)}\left\{S^{k+V^{H}}, A^{k+V^{H}} ; S^{V^{H}}, *\right\}_{W H}^{\circ},
$$

where o means $*, \perp$ or $\nabla$. 
Next, we are going to explain relations between sets of homotopy classes $\left[S^{k+V} ; S^{V}\right]_{G}^{*}$ and $\left[S^{k+V} ; S^{V}\right]_{G}^{\perp}$. Here is our result.

Theorem 1.3. Assume that $(e) \in \operatorname{Iso}(V)$ and $q=\operatorname{dim} G \leq \operatorname{dim} V-k-2$. There is a natural bijection

$$
\mathcal{C}:\left[S^{k+V}, A^{k+V} ; S^{V}, *\right]_{G}^{\perp} \cong\left[S^{q+k+V}, A^{q+k+V} ; S^{V}, *\right]_{G}^{*} .
$$

In the case $k+\operatorname{dim} V^{G} \geq 2$, this is an isomorphism of abelian groups.

The following result on stable equivariant homotopy groups is a consequence of Theorem 1.3.

Theorem 1.4. For every $q \geq 0$ there exists a natural isomorphism

$$
\begin{aligned}
& \bigoplus_{(H) \in \operatorname{Iso}_{q}(V)}\left\{S^{k+V^{H}}, A^{k+V^{H}} ; S^{V^{H}}, *\right\}_{W H}^{\perp} \\
& \longrightarrow \bigoplus_{(H) \in \operatorname{Iso}_{q}(V)}\left\{S^{q+k+V^{H}}, A^{q+k+V^{H}} ; S^{V^{H}}, *\right\}_{W H}^{*},
\end{aligned}
$$

where

$$
\operatorname{Iso}_{q}(V)=\operatorname{Iso}(V) \cap \Phi_{q}(G) .
$$

\section{From homotopy to otopy}

In the proof of Theorem 1.1 certain properties of the so-called equivariant local maps will be crucial (see [2]).

\subsection{Local maps}

Throughout this subsection we assume that $\Omega \subset \mathbb{R}^{k} \oplus V$ is open and $G$ invariant.

A local $G$-equivariant map on $\Omega$ is a pair $(f, U)$ consisting of an open invariant subset $U \subset \Omega$ and an equivariant continuous map $f: U \rightarrow V$ such that $f^{-1}(0)$ is compact. An otopy on $\Omega$ is a pair $(h, \Lambda)$ consisting of an open invariant subset $\Lambda \subset \Omega \times I$ and an equivariant continuous map $h: \Lambda \rightarrow V$ such that $h^{-1}(0)$ is compact.

Denote by $\mathcal{F}_{G}^{*}(\Omega)$ the set of all local $G$-equivariant maps on $\Omega$. We shall write $\mathcal{F}_{G}^{*}[\Omega]$ for the set of otopy classes of local $G$-equivariant maps on $\Omega$. We say that $(f, U) \in \mathcal{F}_{G}^{*}(\Omega)$ is a local $G$-equivariant orthogonal map if

$$
f(x, v) \perp T_{v}(G v)
$$

for each $(x, v) \in U$. The set of all local $G$-equivariant orthogonal maps on $\Omega$ is denoted by $\mathcal{F}_{G}^{\perp}(\Omega)$, and $\mathcal{F}_{G}^{\perp}[\Omega]$ stands for the corresponding set of otopy classes.

A local G-equivariant gradient map is a pair $(f, U) \in \mathcal{F}_{G}^{*}(\Omega)$ such that there are a $G$-invariant open neighbourhood $U^{\prime}$ of $f^{-1}(0), U^{\prime} \subset U$, and a $G$-invariant map $\varphi: U^{\prime} \rightarrow \mathbb{R}$ such that

$$
\nabla_{v} \varphi(x, v)=f(x, v), \quad(x, v) \in U^{\prime} \subset \mathbb{R}^{k} \oplus V .
$$


$\mathcal{F}_{G}^{\nabla}(\Omega)$ denotes the set of all local $G$-equivariant gradient maps on $\Omega$, and $\mathcal{F}_{G}^{\nabla}[\Omega]$ is the corresponding set of otopy classes.

Note that for every local $G$-equivariant $\operatorname{map}(f, U) \in \mathcal{F}_{G}^{\circ}(\Omega)$, where $\circ=$ $*, \perp$ or $\nabla$, there is an otopic local $G$-equivariant map with relatively compact domain. It is enough to take appropriate restriction of $f$. Furthermore, $(f, U)$ can be modified within its otopy class to a local $G$-equivariant map $(\hat{f}, U)$ that is proper. This result was proved in [2] (see Theorem 7.1) in the case $\circ=*$. Cases $\circ=\perp$ and $\circ=\nabla$ can be proved analogously.

With every map $f \in \mathcal{M}_{G}^{\circ}\left(S^{k+V}, S^{V}\right)$ one associates a local $G$-equivariant $\operatorname{map}\left(f_{\mid f^{-1}(V)}, f^{-1}(V)\right) \in \mathcal{F}_{G}^{\circ}\left(\mathbb{R}^{k} \oplus V\right)$. Clearly, the map

$$
f_{\mid f^{-1}(V)}: f^{-1}(V) \longrightarrow V
$$

is proper. Conversely, if $(f, U) \in \mathcal{F}_{G}^{\circ}\left(\mathbb{R}^{k} \oplus V\right)$ and $f$ is proper, then the map $f^{+}: S^{k+V} \rightarrow S^{V}$, defined by

$$
f^{+}(x):= \begin{cases}f(x) & \text { for } x \in U, \\ \infty & \text { for } x \in S^{k+V} \backslash U,\end{cases}
$$

is continuous. Using this observation we will identify $G$-equivariant maps $f \in \mathcal{M}_{G}^{\circ}\left(S^{k+V}, S^{V}\right)$ with proper local $G$-equivariant maps in $\mathcal{F}_{G}^{\circ}\left(\mathbb{R}^{k} \oplus V\right)$.

Theorem 2.1. The assignment $f \mapsto\left(f_{\mid f^{-1}(V)}, f^{-1}(V)\right)$ induces bijections

$$
\mathcal{R}_{1}^{\circ}:\left[S^{k+V}, S^{V}\right]_{G}^{\circ} \approx \mathcal{F}_{G}^{\circ}\left[\mathbb{R}^{k} \oplus V\right]
$$

and

$$
\mathcal{R}_{2}^{\circ}:\left[S^{k+V}, A^{k+V} ; S^{V}, *\right]_{G}^{\circ} \approx \mathcal{F}_{G}^{\circ}\left[\mathbb{R}^{k} \times V_{e}\right],
$$

where $\circ$ means $*, \perp$ or $\nabla$.

The bijection $\mathcal{R}_{1}^{*}$ is proved in [2] (see Theorem 7.1). The other cases can be proved analogously.

\subsection{The splitting lemma}

Throughout this subsection $\Omega$ denotes an open invariant subset of $\mathbb{R}^{k} \oplus V$ and $(H)$ is a maximal isotropy type in $\Omega$. For $x \in \Omega_{(H)}$ we denote by $N_{x}$ the linear subspace of $\mathbb{R}^{k} \oplus V$ defined by $N_{x}:=\left(T_{x} \Omega_{(H)}\right)^{\perp}$. For an open invariant subset $P \subset \Omega_{(H)}$ we consider a vector bundle

$$
\pi: \mathcal{U}(P):=\left\{(x, v) \in P \times V ; v \in N_{x}\right\} \longrightarrow P,
$$

where $\pi(x, v)=x$. For $\varepsilon>0$, we let

$$
\mathcal{U}_{\varepsilon}(P):=\{(x, v) \in \mathcal{U}(P) ;|v|<\varepsilon\} .
$$

Define a map

$$
\nu: \mathcal{U}(P) \longrightarrow \mathbb{R}^{k} \oplus V
$$

by $\nu(x, v):=x+v$.

We say that an open invariant subset $U \subset \Omega$ is $(H)$-normal if there exists $\varepsilon>0$ such that $\nu$ defines a diffeomorphism $\mathcal{U}_{\varepsilon}\left(U_{(H)}\right) \rightarrow U$. Note that if $U$ is $(H)$-normal, then every element of it has a unique representation $x+v$, where $x \in U_{(H)}, v \in N_{x}$. 
Definition 2.2. Let $(H)$ be a maximal element in $\operatorname{Iso}(\Omega)$. We say that $(f, U) \in$ $\mathcal{F}_{G}^{*}(\Omega)$ is $(H)$-normal if $U$ is $(H)$-normal and $x \in U_{(H)}, v \in N_{x}, x+v \in U$ imply $f(x+v)=f(x)+v$. We say that an otopy $(h, \Lambda)$ on $\Omega$ is $(H)$-normal if $\left(h_{t}, \Lambda_{t}\right)$ is $(H)$-normal for all $t \in[0,1]$, where $\Lambda_{t}=\{y \in \Omega ;(y, t) \in \Lambda\}$.

If $\left(f_{1}, U_{1}\right),\left(f_{2}, U_{2}\right) \in \mathcal{F}_{G}^{\circ}(\Omega)$ and $U_{1} \cap U_{2}=\emptyset$, then we put

$$
\left(f_{1}, U_{1}\right) \sqcup\left(f_{2}, U_{2}\right):=\left(f, U_{1} \cup U_{2}\right),
$$

where $f(x)=f_{1}(x)$ for $x \in U_{i}, i=1,2$.

Theorem 2.3 (The splitting principle). Suppose $(H)$ is a maximal element in $\operatorname{Iso}(\Omega)$ and $(f, U) \in \mathcal{F}_{G}^{\circ}(\Omega)$, where $\circ=*, \perp$ or $\nabla$. Then there exist $\left(f_{1}, U_{1}\right),\left(f_{2}, U_{2}\right) \in \mathcal{F}_{G}^{\circ}(\Omega)$ such that

(a) $U_{1} \cap U_{2}=\emptyset, U_{2} \subset \Omega \backslash \Omega_{(H)}$;

(b) $\left(f_{1}, U_{1}\right)$ is $(H)$-normal and $f_{1}(x)=f(x)$ for $x \in U_{1} \cap \Omega_{(H)}$;

(c) $\left(f_{1}, U_{1}\right) \sqcup\left(f_{2}, U_{2}\right)$ and $(f, U)$ represent the same otopy class in $\mathcal{F}_{G}^{\circ}[\Omega]$.

Proof. Without loss of generality, we assume that $U=O_{1} \cup O_{2}$, and

(1) $O_{1}:=\mathcal{U}_{4 \varepsilon}\left(U_{(H)}\right)$, i.e., $O_{1}$ is $(H)$-normal;

(2) $O_{1} \cap O_{2}=\left\{x+v \in O_{1} ; x \in U_{(H)}, 3 \epsilon<|v|<4 \epsilon\right\}$.

Choose a smooth function $\theta: \mathbb{R} \rightarrow \mathbb{R}$ which equals 0 on $(-\infty, 2 \varepsilon]$ and equals 1 on $[3 \varepsilon, \infty)$ and strictly increases on the interval $(2 \varepsilon, 3 \varepsilon)$. Let $r: O_{1} \rightarrow O_{1}$ be defined by $r(x+v):=x+\theta(|v|) v, x \in U_{(H)}, v \in N_{x}$. Define $\widehat{f}: U \rightarrow V$ as follows. In the case $\circ=*$ or $\perp$ we let

$$
\widehat{f}(y):= \begin{cases}f(r(y)) & \text { if } y \in O_{1}, \\ f(y) & \text { if } y \in U \backslash O_{1} .\end{cases}
$$

In the case $\circ=\nabla$ there is $\varphi: U \rightarrow \mathbb{R}$ such that $f=\nabla_{v} \varphi$. We put $\widehat{f}:=\nabla_{v} \widehat{\varphi}$, where

$$
\widehat{\varphi}(y):= \begin{cases}\varphi(r(y)) & \text { if } y \in O_{1}, \\ \varphi(y) & \text { if } y \in U \backslash O_{1} .\end{cases}
$$

In all the three cases, $(f, U)$ and $(\widehat{f}, U)$ are otopic. Replacing deformation $r$ by the family of deformations

$$
r_{t}(x+v):=x+[t \theta(|v|)+(1-t)] v, \quad t \in[0,1],
$$

one obtains an appropriate otopy.

Define $\tilde{f}: U \rightarrow V$,

$$
\widetilde{f}(y):= \begin{cases}\widehat{f}(x)+\eta(|v|) v & \text { if } y=x+v \in O_{1}, \\ \widehat{f}(y) & \text { if } y \in U \backslash O_{1},\end{cases}
$$

where $\eta: \mathbb{R} \rightarrow \mathbb{R}$ is a smooth function which equals 1 on $(-\infty, \varepsilon]$ and equals 0 on $[2 \varepsilon, \infty)$ and strictly decreases on the interval $(\varepsilon, 2 \varepsilon)$. In the case $\circ=\nabla$ we need $\eta(|v|) v$ to be a gradient map. Choose a smooth function $\alpha: \mathbb{R} \rightarrow \mathbb{R}$ which equals 1 on $\left(-\infty, \frac{1}{2} \varepsilon^{2}\right]$ and equals 0 on $\left[2 \varepsilon^{2}, \infty\right)$ and strictly decreases on the interval $\left(\frac{1}{2} \varepsilon^{2}, 2 \varepsilon^{2}\right)$. Let $\beta(t):=\int_{0}^{t} \alpha(\tau) d \tau$ and $\psi(v)=\beta\left(\frac{1}{2}|v|^{2}\right)$. Then $\nabla \psi(v)=\alpha\left(\frac{1}{2}|v|^{2}\right) v=: \eta(|v|) v$. 
Finally, let $U_{1}:=\left\{x+v \in O_{1} ; x \in U_{(H)},|v|<1\right\}, U_{2}:=U \backslash \overline{U_{1}}$. The local maps $\left(f_{1}, U_{1}\right)=\left(\widetilde{f}_{\mid U_{1}}, U_{1}\right)$ and $\left.\left(f_{2}, U_{2}\right)=\left(\widehat{f}_{\mid U_{2}}\right), U_{2}\right)$ satisfy conditions (a), (b) and (c).

Let $(f, U) \in \mathcal{F}_{G}^{\circ}(\Omega)$ and let $\left(f_{1}, U_{1}\right),\left(f_{2}, U_{2}\right)$ be as in Theorem 2.3. Let $U_{0}:=U_{1} \cap V^{H}, f_{0}(x):=f_{1}(x)$ for $x \in U_{0}$. The otopy class of a local map $(f, U) \in \mathcal{F}_{G}^{\circ}(\Omega)$ is denoted by $[f, U]^{\circ}$. Assigning to $[f, U]^{\circ} \in \mathcal{F}_{G}^{\circ}[\Omega]$ the pair $\left(\left[f_{0}, U_{0}\right]^{\circ},\left[f_{2}, U_{2}\right]^{\circ}\right)$, we obtain the bijection

$$
\mathcal{A}_{\Omega, H}: \mathcal{F}_{G}^{\circ}[\Omega] \longrightarrow \mathcal{F}_{W H}^{\circ}\left[\Omega_{H}\right] \times \mathcal{F}_{G}^{\circ}\left[\Omega \backslash \Omega_{(H)}\right] .
$$

\subsection{Proof of Theorem 1.1}

Let

$$
\operatorname{Iso}\left(\mathbb{R}^{k} \oplus V\right)=\left\{\left(H_{1}\right),\left(H_{2}\right), \ldots,\left(H_{p}\right)\right\},
$$

where $\left(H_{j}\right) \geq\left(H_{i}\right)$ (i.e., $H_{i}$ is conjugate to a subgroup of $H_{j}$ ) implies $j \leq i$.

Set $\Omega_{1}:=\mathbb{R}^{k} \oplus V$ and define

$$
\Omega_{i+1}:=\Omega_{i} \backslash\left(\Omega_{i}\right)_{\left(H_{i}\right)}, \quad i=1, \ldots, p-1 .
$$

Note that Iso $\left(\Omega_{i}\right)=\left\{\left(H_{i}\right),\left(H_{i+1}\right), \ldots,\left(H_{p}\right)\right\}$. Using (2.1) we have bijections

$$
\mathcal{A}_{i}:=\mathcal{A}_{\Omega_{i}, H_{i}}: \mathcal{F}_{G}^{\circ}\left[\Omega_{i}\right] \longrightarrow \mathcal{F}_{W H_{i}}^{\circ}\left[\Omega_{H_{i}}\right] \times \mathcal{F}_{G}^{\circ}\left[\Omega_{i+1}\right]
$$

for $i=1, \ldots, p-1$. If $a \in \mathcal{F}_{G}^{\circ}\left[\Omega_{1}\right]$, then

$$
\mathcal{A}_{1}(a)=\left(a_{1}, b_{2}\right) \in \mathcal{F}_{W H_{1}}^{\circ}\left[\left(\mathbb{R}^{k} \oplus V^{H_{1}}\right)_{e}\right] \times \mathcal{F}_{G}^{\circ}\left[\Omega_{2}\right] .
$$

For $i=2, \ldots, c p-1$ one has

$$
\mathcal{A}_{i}\left(b_{i}\right)=\left(a_{i}, b_{i+1}\right) \in \mathcal{F}_{W H_{i}}^{\circ}\left[\left(\mathbb{R}^{k} \oplus V^{H_{i}}\right)_{e}\right] \times \mathcal{F}_{G}^{\circ}\left[\Omega_{i+1}\right] .
$$

Define

$$
a_{p}:=b_{p} \in \mathcal{F}_{G}^{\circ}\left[\Omega_{p}\right]=\mathcal{F}_{W H_{p}}^{\circ}\left[\left(\mathbb{R}^{k} \oplus V^{H_{p}}\right)_{e}\right]
$$

and put

$$
\mathcal{A}:=\left(a_{1}, a_{2}, \ldots, a_{p}\right) \in \mathcal{F}_{W H_{1}}^{\circ}\left[\left(\mathbb{R}^{k} \oplus V^{H_{1}}\right)_{e}\right] \times \cdots \times \mathcal{F}_{W H_{p}}^{\circ}\left[\left(\mathbb{R}^{k} \oplus V^{H_{p}}\right)_{e}\right] .
$$

Now the assertion is a consequence of Theorem 2.1.

\subsection{Proof of Theorem 1.2}

Let $V \subset W=V \oplus \mathbb{R}^{n}$ be representations of a compact Lie group $G$.

Notice that

$$
\operatorname{Iso}(V)=\operatorname{Iso}(W) .
$$

The following remark is a direct consequence of (2.3) and the definition of $\mathcal{A}_{\Omega, H}$. 
Remark 2.4. Assume that $\Omega \subset \mathbb{R}^{k} \oplus V$ is a $G$-invariant open set and $(H)$ is a maximal orbit type in $\operatorname{Iso}(\Omega)$. Then $(H)$ is a maximal orbit type in Iso $\left(\Omega \times \mathbb{R}^{n}\right)$ and the following diagram commutes

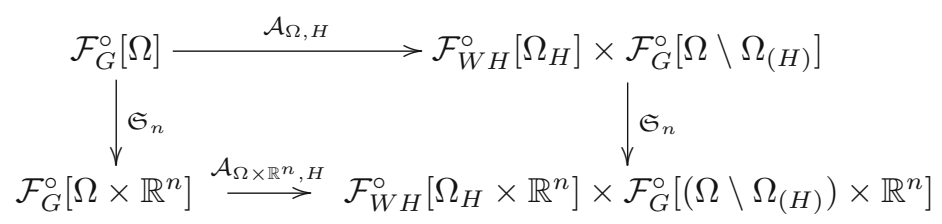

Our theorem follows from the above remark and Theorem 1.1.

\section{Proof of Theorems 1.3 and 1.4}

In this section we will need some results concerning fiberwise homotopy theory. For the notation and terminology we refer the reader to [5].

Throughout this section, $V$ is a fixed real orthogonal $n$-dimensional representation of a compact Lie group $G$ such that $V_{e} \neq \emptyset$. We fix a nonnegative integer $k$ and assume that $\operatorname{dim}\left(\mathbb{R}^{k} \oplus V^{G}\right) \geq 2$. Note that $\left(\mathbb{R}^{k} \oplus V\right)_{e}$ is open and dense in $\mathbb{R}^{k} \oplus V$. Put $M:=\left(\mathbb{R}^{k} \oplus V\right)_{e} / G$.

\subsection{From equivariant to fiberwise maps}

Let

$$
E:=V \times \mathbb{R}^{k} \times V_{e} \longrightarrow \mathbb{R}^{k} \times V_{e}
$$

denote the product $G$-bundle. Identifying the tangent space $T_{v} V$ at $v \in V$ with $V$ define $N_{v}:=\left(T_{v}(G v)\right)^{\perp}$. Then

$$
\nu: N:=\left\{(w, x, v) \in V \times \mathbb{R}^{k} \times V_{e} ; w \in N_{v}\right\} \longrightarrow \mathbb{R}^{k} \times V_{e}
$$

is a vector $G$-bundle, where $\nu(w, x, v)=(x, v)$. Let

$$
E^{+}, N^{+} \longrightarrow \mathbb{R}^{k} \times V_{e}
$$

be fiberwise one-point compactifications of $E$ and $N$, respectively.

Dividing out $G$-action, one gets the corresponding quotient bundles

$$
\mathcal{E} \longrightarrow M \text { and } \mathcal{N} \longrightarrow M \text {. }
$$

Suppose that $f: S^{q+k+V} \rightarrow S^{V}$ is a $G$-equivariant map satisfying

$$
\overline{f^{-1}(V)} \Subset \mathbb{R}^{q} \times \mathbb{R}^{k} \times V_{e} .
$$

Let $\widehat{\xi}: S^{q} \times \mathbb{R}^{k} \times V_{e} \rightarrow E^{+}$be defined by

$$
\widehat{\xi}_{(x, v)}(y):= \begin{cases}(f(y, x, v), x, v) & \text { if }(y, x, v) \in f^{-1}(V), \\ (*,(x, v)) & \text { if } f(y, x, v)=* .\end{cases}
$$

Clearly $\widehat{\xi}$ is a fiberwise $G$-map over $\mathbb{R}^{k} \times V_{e}$ from the product bundle $S^{q} \times$ $\mathbb{R}^{k} \times V_{e} \rightarrow \mathbb{R}^{k} \times V_{e}$ into $E^{+}$. 
Dividing out this $G$-action, one obtains the bundle map

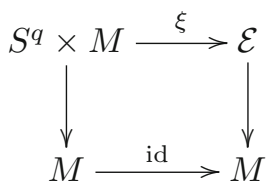

Since $\xi$ is compactly supported, the homotopy class of $\xi$ determines an element in ${ }_{c} \pi_{M}^{0}\left[S^{q} \times M ; \mathcal{E}\right]$.

Consequently, the following map is defined:

$$
\mathcal{B}^{*}:\left[S^{q+k+V}, A^{q+k+V} ; S^{V}, *\right]_{G}^{*} \longrightarrow{ }_{c} \pi_{M}^{0}\left[S^{q} \times M ; \mathcal{E}\right] .
$$

It is easily seen that $\mathcal{B}^{*}$ is a bijection.

Suppose now that $g: S^{k+V} \rightarrow S^{V}$ is an orthogonal equivariant map satisfying

$$
\overline{g^{-1}(V)} \Subset \mathbb{R}^{k} \times V_{e} .
$$

Let $\widehat{\eta}: S^{0} \times \mathbb{R}^{k} \times V_{e} \rightarrow \mathbb{R}^{k} \times V_{e}$ be given by

$$
\widehat{\eta}_{(x, v)}(y):= \begin{cases}(g(x, v), x, v) & \text { if }(x, v) \in g^{-1}(V) \text { and } y \neq *, \\ (*,(x, v)) & \text { if } g(x, v)=* \text { or } y=* .\end{cases}
$$

Clearly $\widehat{\eta}$ is a fiberwise $G$-map over $\mathbb{R}^{k} \times V_{e}$ from the product bundle $S^{0} \times$ $\mathbb{R}^{k} \times V_{e} \rightarrow \mathbb{R}^{k} \times V_{e}$ into $N^{+}$.

Dividing out $G$-action, one gets the bundle map

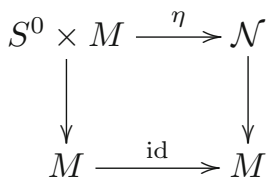

This way one defines the bijection

$$
\mathcal{B}^{\perp}:\left[S^{k+V}, A^{k+V} ; S^{V}, *\right]_{G}^{\perp} \longrightarrow{ }_{c} \pi_{M}^{0}\left[S^{0} \times M ; \mathcal{N}\right] .
$$

\subsection{Proof of Theorem 1.3}

One easily shows that $R^{k} \times V_{e}$ admits $q=\operatorname{dim} G$ linearly independent fundamental vector fields (see [1, p. 14]). Consequently, $\mathcal{E} \approx \mathcal{R} \wedge \mathcal{N}$, where $\mathcal{R}$ stands for a product bundle $S^{q} \times M \rightarrow M$. By [5, Proposition 3.18] the right vertical arrow in the commutative diagram

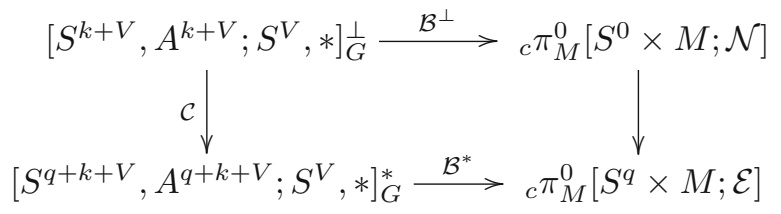

represents an isomorphism. Therefore, the left vertical arrow in this diagram defines an isomorphism $\mathcal{C}$ as claimed. 


\section{References}

[1] M. Audin, The Topology of Torus Actions on Symplectic Manifolds. Progress in Mathematics 93, Birkhäuser Verlag, Basel, 1991.

[2] P. Bartłomiejczyk, K. Gȩba and M. Izydorek, Otopy classes of equivariant maps. J. Fixed Point Theory Appl. 7 (2010), 145-160.

[3] J. C. Becker and D. H. Gottlieb, Vector fields and transfers. Manuscripta Math. 72 (1991), 111-130.

[4] G. E. Bredon, Introduction to Compact Transformation Groups. Academic Press, New York, 1972.

[5] M. C. Crabb and I. James, Fibrewise Homotopy Theory. Springer-Verlag London Ltd., London, 1998.

[6] E. N. Dancer, A new degree for $S^{1}$-invariant gradient mappings and applications. Ann. Inst. H. Poincaré Anal. Non Linéaire 2 (1985), 329-370.

[7] E. N. Dancer, K. Gęba and S. Rybicki, Classification of homotopy classes of equivariant gradient maps. Fund. Math. 185 (2005), 1-18.

[8] H. Hauschild, Äquivariante Homotopie. I. Arch. Math. (Basel) 29 (1977), 158165 (in German).

[9] L. G. Lewis, Jr., J. P. May and M. Steinberger, Equivariant Stable Homotopy Theory. Lecture Notes in Math. 1213, Springer-Verlag, Berlin, 1986.

[10] W. Marzantowicz and C. Prieto, A decomposition formula for equivariant stable homotopy classes. Topol. Methods Nonlinear Anal. 33 (2009), 285-292.

[11] T. tom Dieck, The Burnside ring of a compact Lie group. I. Math. Ann. 215 (1975), 235-250.

[12] T. tom Dieck, Transformation Groups. de Gruyter Stud. Math. 8, de Gruyter, Berlin, 1987.

[13] T. tom Dieck, Representation Theory. http://www.uni-math.gwdg.de/tammo/ rep.pdf, 2009.

Kazimierz Gȩba

Faculty of Technical Physics and Mathematics

Gdańsk University of Technology

Narutowicza 11/12

80-952 Gdańsk, Poland

e-mail: geba@mifgate.mif.pg.gda.pl

Marek Izydorek

Faculty of Technical Physics and Mathematics

Gdańsk University of Technology

Narutowicza 11/12

80-952 Gdańsk, Poland

e-mail: izydorek@mifgate.mif.pg.gda.pl

Open Access This article is distributed under the terms of the Creative Commons Attribution License which permits any use, distribution, and reproduction in any medium, provided the original author(s) and the source are credited. 